\title{
The association between Medicare Advantage market penetration and diabetes in the United States
}

\begin{abstract}
Steven W. Howard ${ }^{1 *}$, Stephanie Lazarus Bernell' ${ }^{2}$, Jennifer Wilmott ${ }^{3}$, M. Faizan Casim ${ }^{4}$, Jing Wang ${ }^{3}$, Lindsey Pearson ${ }^{5}$, Caitlin M. Byler ${ }^{6}$ and Zidong Zhang ${ }^{7}$

${ }^{1}$ Health Management and Policy, Center for Outcomes Research, Saint Louis University, St. Louis, MO, USA, ${ }^{2}$ Health Policy and Management, School of Social and Behavioral Health Services, Oregon State University, Corvallis, OR, USA,

${ }^{3}$ Biostatistics, Saint Louis University, St. Louis, MO, USA, ${ }^{4}$ HealthCom Research and Solutions, Inc., Fredericksburg, VA, USA, ${ }^{5}$ Saint Luke's Health System, Kansas City, MO, USA, ${ }^{6}$ The University of Texas MD Anderson Cancer Center, Houston, TX, USA, ' Jefferson County Health Department, Hillsboro, MO, USA
\end{abstract}

The objective of this study is to explore the extent to which managed care market penetration in the United States is associated with the presence of chronic disease. Diabetes was selected as the chronic disease of interest due to its increasing prevalence as well as the disease management protocols that can lessen disease complications. We hypothesized that greater managed care market penetration would be associated with (1)

Edited by:

Edward Broughton,

University Research Co., LLC, USA

Reviewed by:

Tamar A. Klaiman,

University of the Sciences in

Philadelphia, USA

Moira J. McKinnon,

Royal Australian College of

Physicians, Australia

${ }^{*}$ Correspondence:

Steven W. Howard

showar19@SLU.edu

Specialty section:

This article was submitted to Public

Health Policy, a section of the

journal Frontiers in Public Health

Received: 02 July 2015 Accepted: 22 September 2015

Published: 08 October 2015

Citation:

Howard SW, Bernell SL, Wilmott J, Casim MF, Wang J, Pearson L, Byler CM and Zhang Z (2015) The association between Medicare Advantage market penetration and diabetes in the United States.

Front. Public Health 3:229. doi: 10.3389/fpubh.2015.00229 lower prevalence of diabetes and (2) lower prevalence of diabetes-related comorbidities (DRCs) among diabetics. Data for this analysis came from two sources. We merged Medicare Advantage (MA) market penetration data from the Centers for Medicare and Medicaid Services (CMS) with data from the Medical Expenditure Panel Survey (MEPS) (2004-2008). Results suggest that county-level MA market penetration is not significantly associated with prevalence of diabetes or DRCs. That finding is quite interesting in that managed care market penetration has been shown to have an effect on utilization of inpatient services. It may be that managed care protocols do not offer the same benefits beyond the inpatient setting.

Keywords: cardiometabolic conditions, diabetes, cardiovascular disease, MEPS, Medicare Advantage, market penetration, spillover effects

\section{Introduction}

Over the past 15 years, there has been a marked increase in the number of Medicare beneficiaries enrolled in managed care plans. Medicare Advantage (MA) enrollment among Medicare beneficiaries has rapidly increased from $9 \%$ in 1995 to $30 \%$ in $2014(1,2)$. The market penetration rate of MA varies from state to state and county to county, with urban areas having higher enrollment rates (26\%) compared to rural areas $(15 \%)(3,4)$.

Research on the effects of managed care penetration has primarily focused on inpatient care and prevention services. Results indicate that increased managed care market penetration is linked to higher rates of vaccinations and disease screenings among the general population (5-7). Greater managed care market penetration is also associated with reduced utilization of unnecessary inpatient procedures among Medicare beneficiaries $(8,9)$ reductions in inpatient complications $(10)$ and reduced mortality rates after hospital discharge $(11,12)$. 
The spillover effect of managed care processes is also of great interest. Work in this area suggests that strategies implemented by health care providers contracted with managed care organizations (MCOs) spillover to those providers' patients who are not members of MCOs, particularly in areas of high MCO market penetration $(5,6,9,10)$. If spillover effects are truly impactful, it is reasonable to posit that in areas with high MCO market penetration, physicians will identify chronic conditions more quickly and prescribe treatment (and disease management), which may lead to slower progression of disease $(5,9,13-17)$.

The objective of this study is to explore the extent to which managed care market penetration in the United States is associated with the presence of chronic disease. Diabetes was selected as the chronic disease of interest due to its increasing prevalence as well as the disease management protocols that can lessen disease complications. We hypothesized that greater managed care market penetration would be associated with (1) lower prevalence of diabetes and (2) lower prevalence of diabetes-related comorbidities (DRCs) among diabetics.

Diabetes mellitus (DM) affects nearly 29.1 million (9.3\%) people in the United States, and costs the nation an estimated $\$ 245$ billion annually (18). More than one in four Americans over the age of 65 have DM, and their morbidity contributes to the financial strain on the Medicare and Medicaid programs $(18,19)$.

Improperly treated DM can result in elevated risk for other DRCs, including vascular disease, neuropathy, renal disease, retinopathy, and cardiovascular complications, such as stroke, heart disease, coronary artery disease, and myocardial infarction. Compared to a non-diabetic, an individual with DM has a two to fourfold greater risk of dying from heart disease or having a stroke (18).

Type 2 diabetes is often used as an example of a chronic condition that can benefit from disease management tools often used by managed care (20-22). When effectively implemented, disease management protocols have reduced the rate of diabetes and associated DRCs (23-30).

\section{Materials and Methods}

The data for this study were drawn from the Agency for Healthcare Research and Quality's (AHRQ) Medical Expenditure Panel Survey (MEPS) (31) and the Centers for Medicare and Medicaid Services (CMS). CMS provided the MA market penetration data $(32,33)$. MA market penetration is calculated by taking the number of MA enrollees in a county or state divided by the number of Medicare beneficiaries in the same area. We merged MA market penetration data with data for all Medicare beneficiaries in MEPS from 2004 to 2008 (panels 9-12), based on each beneficiary's county of residence.

The MEPS household component (MEPS-HC) is a nationally representative survey of non-institutionalized individuals residing in households sampled from the previous year's National Health Interview Survey. The individuals followed in MEPS are grouped into panels and surveyed five times (rounds) over 2 years. A new panel begins each year, resulting in the overlapping panel design (one panel's first year is concurrent with the previous panel's second year) (31). After university institutional review board approval, we used data from the non-public MEPS-HC (via AHRQ's secure data center), including information on individuals' and households' demographics (including county and state of residence), diagnoses, and coverage by Medicare and other insurance.

In addition to standard exploratory data analysis, two multivariate logistic regression models were used to explore the association between MA market penetration, diabetes, and DRCs at the individual level. The key independent variable of interest, countylevel MA market penetration, was categorized into three groups: $<12.5,12.5-24.9 \%$, and $\geq 25 \%$. In establishing these groupings, we considered previous research by Baker et al. (5) and Bundorf et al. (8) with upward adjustment of the lower and middle categories to account for the growth in MA market penetration nationwide since the date of these publications.

Individual-level characteristics include race, gender, age, level of education, income as a percent of federal poverty level (FPL), urban residence (in a metropolitan statistical area), having other insurance in addition to Medicare, and having a managed care plan $(34,35)$.

In the first model, exploring the relationship between diabetes, DRCs and MA market penetration, we retained the full sample of 8089 Medicare beneficiaries in the 2004-2008 MEPS (panels 9-12). The second model evaluates the association between MA market penetration and the prevalence of DRCs among diabetic beneficiaries. In this model, individuals were selected who reported diabetes at the end of the first $(n=1761)$ or second $(n=1926)$ years of their panel. DRCs were defined using the priority conditions in MEPS and included hypertension, coronary heart disease (CHD), stroke or transient ischemic attack (TIA), high cholesterol, diabetes-related eye or kidney disease, angina, or heart attack $(36,37)$.

Survey procedures were used to allow for the complex sampling design of MEPS. We used weights provided by MEPS to ensure that the data were representative of the US civilian, noninstitutionalized Medicare population at the time the data were collected. The weights were applied in both the descriptive analysis and the logistic regression model, to adjust for non-response and attrition, and in order to make estimates of person-level changes in selected variables.

\section{Results}

\section{Descriptive Analysis}

The population for this study included 8089 Medicare beneficiaries who participated in MEPS between 2004 and 2008 (panels 9-12). The data had roughly equal representation from each of the four panels. Women made up $56.6 \%$ of the sample, and $53 \%$ were married (Table 1). Nearly $85 \%$ were identified as White, and only $7 \%$ were Hispanic. Nearly one in five were under the age of $65(18.8 \%)$, which is slightly more than the national average $(17 \%)$, and one in five were at least 80 years of age. One-third resided in a household with income higher than $400 \%$ of FPL. While more than $25 \%$ did not receive a high-school education, $20 \%$ of the sample had a college education or greater. Nearly $80 \%$ of respondents lived in urban areas (as defined by the US Census Bureau). Slightly more than half had some insurance coverage 
TABLE 1 | Descriptive statistics. ${ }^{b}$

\begin{tabular}{|c|c|c|c|c|c|}
\hline Variable & $N$ & Percent & Variable & $N$ & Percent \\
\hline Age groups & & & Education & & \\
\hline$<50$ & 612 & $6.48 \%$ & Less than high school & 2715 & $25.86 \%$ \\
\hline $50-64$ & 1123 & $12.40 \%$ & High school & 2613 & $35.48 \%$ \\
\hline $65-69^{a}$ & 1922 & $24.14 \%$ & Some college & 1282 & $18.42 \%$ \\
\hline $70-74$ & 1566 & $19.27 \%$ & 4 years college & 744 & $11.40 \%$ \\
\hline $75-79$ & 1302 & $17.07 \%$ & $>4$ years college ${ }^{a}$ & 579 & $8.84 \%$ \\
\hline $80-84$ & 936 & $12.40 \%$ & Marital status & & \\
\hline$>85$ & 628 & $8.24 \%$ & Married $^{\mathrm{a}}$ & 4068 & $53.00 \%$ \\
\hline Gender & & & Managed care organization & & \\
\hline Female $^{a}$ & 4616 & $56.62 \%$ & Had MCO at the end of first year ${ }^{a}$ & 1268 & $14.14 \%$ \\
\hline Race & & & Other insurance & & \\
\hline White $^{\mathrm{a}}$ & 6287 & $84.77 \%$ & Had other insurance ${ }^{a}$ & 3574 & $51.27 \%$ \\
\hline Hispanic & & & MA market penetration & & \\
\hline Not Hispanic ${ }^{a}$ & 7032 & $92.80 \%$ & $<12.5 \%$ & 4741 & $57.70 \%$ \\
\hline Income (\% FPL) & & & 12.5 to $<25 \%$ & 1218 & $16.47 \%$ \\
\hline$<100 \% \mathrm{FPL}$ & 1535 & $13.54 \%$ & $25 \%$ or more ${ }^{a}$ & 2130 & $25.83 \%$ \\
\hline $100-125 \%$ & 684 & $6.84 \%$ & & & \\
\hline $126-200 \%$ & 1458 & $17.27 \%$ & Panel & & \\
\hline $201-399 \%$ & 2186 & $29.02 \%$ & Panel 9 (2004-2005) & 2017 & $24.37 \%$ \\
\hline$>400 \% \mathrm{FPL}^{\mathrm{a}}$ & 2222 & $33.33 \%$ & Panel 10 (2005-2006) & 2115 & $24.37 \%$ \\
\hline Urban/rural & & & Panel 11 (2006-2007) & 2283 & $25.30 \%$ \\
\hline Urban $^{a}$ & 6248 & $79.28 \%$ & Panel 12 (2007-2008) & 1674 & $25.96 \%$ \\
\hline
\end{tabular}

${ }^{a}$ Reference groups.

${ }^{b}$ All percent statistics are weighted. $N$ is unweighted.

besides Medicare. Fourteen percent had coverage through MCOs. Among Medicare beneficiaries, one in four resided in counties with high MA market penetration $(\geq 25 \%)$, and one in six resided in areas of moderate MA market penetration (12.5-25\% county penetration).

\section{Multivariate Logistic Regression Results}

The main independent variable of interest is MA market penetration. The results suggest that market penetration of managed care (in the respondent's county of residence) is not significantly associated with individual-level diabetes or DRCs. Respondent's insurance type (private insurance in addition to Medicare, and/or more specifically managed care membership) is also not significantly associated with diabetes or DRCs (Table 2).

Factors that appear to be significantly associated with diabetes and DRC prevalence include race, reduced income, age, and place of residence. The only variable in the weighted, multivariate model that is significantly associated with DRCs among diabetics is place of residence. Living in a rural area is significantly associated with a reduced likelihood of diabetes and DRCs among the overall MEPS Medicare beneficiary group.

However, rurality is strongly associated with an increased likelihood of DRCs among diabetics. It may be that rural diabetics have reduced access to specialty care, resulting in a greater likelihood of DRCs.

The odds of having diabetes or a DRC increase with age and for non-White individuals. Compared to respondents with household incomes $\geq 400 \%$ of FPL, a lower level of income increases the odds of diagnosis. Although a significant relationship was observed between diagnosis and income, no statistically significant relationship was observed between diagnosis and education.
Our analysis relies on publically available data and uses fairly straightforward methods. It may be the case that in order to tease out the effect that MA market penetration has on diabetes a different type of empirical analysis is needed. To this end, we developed an alternative model that examined the association between MA market penetration and the occurrence of new cases of diabetes among Medicare beneficiaries between year $1(n=1761)$ and year $2(n=1926)$. We excluded 1761 respondents who reported having diabetes at the end of their first year in the MEPS panel. This resulted in retention of $6328(79.2 \%)$ of our original observations. Like the other models in Table 2, the managed care market penetration variable was not a significant factor in the likelihood of being newly diagnosed with diabetes (results not shown in this paper). The one interesting finding from this additional model was that compared to beneficiaries between the ages of 65 and 70 , respondents age 85 or older had less than half the odds of being diagnosed with diabetes. This lower incidence of diabetes in the oldest group of Medicare beneficiaries may be a survivor effect $(38,39)$.

\section{Discussion}

In this study, we examined the associations between MA market penetration and Medicare beneficiaries' (1) diabetes and DRC diagnoses overall and (2) DRCs among diabetics. This analysis is one of the first since the passage of the Medicare Modernization Act to address the role that market penetration of MA may have on chronic disease and disease progression.

Although the empirical results do not support our hypotheses, this study is important on two fundamental levels. The first relates to whether "non-significant results" can tell a story. While our results are not statistically significant, that finding in and of itself 


\begin{tabular}{|c|c|c|}
\hline & \\
\hline & $\begin{array}{l}\text { Prevalence of diabetes and } \\
\text { diabetes-related comorbidities (DRCs) }(n=8089)\end{array}$ & $\begin{array}{l}\text { Prevalence of DRCs } \\
\text { among diabetics }(n=1926)\end{array}$ \\
\hline Market penetration $<12.5 \%$ & 1.19 & 0.996 \\
\hline Non-White & $1.258^{\star \star}$ & 1.878 \\
\hline Hispanic & 1.181 & 1.26 \\
\hline Federal poverty level (FPL) <100\% & $1.312 *$ & 0.964 \\
\hline FPL 201-399\% & $1.234 *$ & 1.334 \\
\hline Not married & 0.987 & 1.47 \\
\hline Male & 0.94 & 0.737 \\
\hline Less than high-school degree & 1.195 & 1.541 \\
\hline High-school degree & 1.162 & 0.722 \\
\hline Some college & 1.199 & 1.414 \\
\hline 4 years of college & 0.975 & 0.663 \\
\hline Age 80-84 & $1.625^{\star \star \star}$ & 1.836 \\
\hline Age $85+$ & $1.696^{\star \star \star}$ & 1.167 \\
\hline No MCO & 1.028 & 1.192 \\
\hline No other insurance & 0.933 & 0.647 \\
\hline Not in MSA & $0.787^{\star \star}$ & $2.867^{\star \star}$ \\
\hline
\end{tabular}

${ }^{*} p<0.10 ;{ }^{* *} p<0.05 ;{ }^{* * *} p<0.01$.

${ }^{a}$ All statistics are weighted.

is substantively significant. It is possible that the lack of association between market penetration and diabetes is an indication that managed care is not working as well as previous research has indicated. It could also be the case that there are limited spillover effects of managed care. Lastly, it could be that the protocols are so varied in scope and implementation that no systematic impact can be detected (i.e., more direct measures of the type and longevity of disease management protocols within the individual plans). The second contribution is methodological. We suggest researchers use our study as a springboard for future work.

\section{Limitations}

While this study had unique strengths, there were limitations. Screening rates influence the prevalence rates of diagnosed disease. Diabetes screening rates for this population were unavailable. Since screening rates are known to increase under managed care (5-7), we expect that there is higher prevalence of screening in areas of higher managed care market penetration, all other things being constant. In the absence of a systemwide model, the effect of changes in screening procedures and rates on diabetes incidence and complications cannot be fully ascertained.

We found significant results for rural Medicare beneficiaries. However, given that most rural areas in the United States are primary care-shortage areas, it is possible that the rate of undiagnosed diabetes and DRCs is higher in rural areas, and the prevalence of diagnosed disease lower. The modest number of rural Medicare beneficiaries in MEPS makes it difficult to understand the impact of managed care market penetration. Future work will include a larger population of rural individuals, and will focus on the relationships between rurality and chronic disease.

The MEPS survey data are self-reported. With self-report data, MEPS is subject to recall error and the honesty of participants' responses to surveyors.

At a minimum, new work on the effects of managed care (or specifically MA) market penetration on chronic diseases, such as diabetes, should include the following:

(1) Representative data from all United States counties. In our study, only a sample of United States counties is represented in each MEPS panel. If these counties (or those not sampled) are outliers on the distribution of MA market penetration, this study's findings may not be indicative of the true national trends.

(2) Clinical data. Due to data limitations, our study relied on the use of household-reported health information, not clinical data. Clinical or medical claims data may reduce any problems of recall bias and provide more precise diagnoses. In addition, clinical data may be able to capture differences in clinical severity of the underlying diagnoses and enable researchers to consider the individual's overall "disease portfolio."

(3) At least 4 years of longitudinal data. In our study, disease diagnosis variables were limited primarily to the priority conditions questions in MEPS, which are asked only at the end of each year (rounds 3 and 5), leaving only 1 year for new diagnoses to be reported.

On balance, we do not take our results as the definitive answer to the prevailing question: Does MA market penetration 
impact disease progression? Given that MA market penetration is at $30 \%$, and growing each year, future work in this area is essential (40). The Affordable Care Act and various state reform efforts call for innovative healthcare delivery models that bear similarities to

\section{References}

1. Kaiser Family Foundation. Medicare Advantage Fact Sheet. (2014). Available from: http://kff.org/medicare/fact-sheet/medicare-advantage-fact-sheet/

2. Medicare Payment Advisory Commission. A Data Book: Health Care Spending and the Medicare Program. Washington, DC: Medicare Payment Advisory Commission (2012).

3. McDowell A, Sheingold S. Payment for Medicare Advantage Plans: Policy Issues and Options. (2009). Available from: http://aspe.hhs.gov/health/reports/09/ medicareadvantage/index.shtml

4. Kaiser Family Foundation. Medicare Advantage Fact Sheet. (2011). Available from: http://familiesusa2.org/conference/health-action-2012/toolkit/content/ pdfs/medicare-adv.pdf

5. Baker LC, Phillips KA, Haas JS, Liang SY, Sonneborn D. The effect of area HMO market share on cancer screening. Health Serv Res (2004) 39(6 Pt 1):1751-72. doi:10.1111/j.1475-6773.2004.00316.x

6. Gillies RR, Chenok KE, Shortell SM, Pawlson G, Wimbush JJ. The impact of health plan delivery system organization on clinical quality and patient satisfaction. Health Serv Res (2006) 41(4 Pt 1):1181-99. doi:10.1111/j.14756773.2006.00529.x

7. Scanlon DP, Swaminathan S, Chernew M, Lee W. Market and plan characteristics related to HMO quality and improvement. Med Care Res Rev (2006) 63(6 Suppl):56S-89S. doi:10.1177/1077558706293835

8. Bundorf MK, Schulman KA, Stafford JA, Gaskin D, Jollis JG, Escarce JJ. Impact of managed care on the treatment, costs, and outcomes of fee-for-service Medicare patients with acute myocardial infarction. Health Serv Res (2004) 39(1):131-52. doi:10.1111/j.1475-6773.2004.00219.x

9. Meara E, Landrum MB, Ayanian JZ, McNeil BJ, Guadagnoli E. The effect of managed care market share on appropriate use of coronary angiography among traditional Medicare beneficiaries. Inquiry (2004) 41(2):144-58. doi:10.5034/ inquiryjrnl_41.2.144

10. Sari N. Do competition and managed care improve quality? Health Econ (2002) 11(7):571-84. doi:10.1002/hec.726

11. Escarce JJ, Jain AK, Rogowski J. Hospital competition, managed care, and mortality after hospitalization for medical conditions: evidence from three states. Med Care Res Rev (2006) 63(6 Suppl):112S-40S. doi:10.1177/ 1077558706293839

12. Rogowski J, Jain AK, Escarce JJ. Hospital competition, managed care, and mortality after hospitalization for medical conditions in California. Health Serv Res (2007) 42(2):682-705. doi:10.1111/j.1475-6773.2006.00631.x

13. Baker LC. The effect of HMOs on fee-for-service health care expenditures: evidence from Medicare. J Health Econ (1997) 16(4):453-81. doi:10.1016/S01676296(96)00535-8

14. Barrett, D. P. (2009). Personal Interview.

15. Berenson RA. Medicare disadvantaged and the search for the elusive level playing field'. Health Aff (Millwood) (2004):W4-572-585. doi:10.1377/hlthaff. w4.572

16. Chernew M, Decicca P, Town R. Managed care and medical expenditures of Medicare beneficiaries. J Health Econ (2008) 27(6):1451-61. doi:10.1016/j. jhealeco.2008.07.014

17. Nicholas LH. Does Managed Care Reduce Federal Spending? Evidence from Medicare. Ann Arbor, MI: Population Studies Center, University of Michigan, Institute for Social Research (2009).

18. Centers for Disease Control and Prevention. National Diabetes Statistics Report. (2014). Available from: http://www.cdc.gov/diabetes/pubs/statsreport14/ national-diabetes-report-web.pdf

19. Soe K, Sacerdote A, Karam J, Bahtiyar G. Management of type 2 diabetes mellitus in the elderly. Maturitas (2011) 70(2):151-9. doi:10.1016/j.maturitas. 2011.07.006

20. Dall TM, Roary M, Yang W, Zhang S, Chen YJ, Arday DR, et al. Health care use and costs for participants in a diabetes disease management program, United States, 2007-2008. Prev Chronic Dis (2011) 8(3):A53. managed care. Understanding the role of MA market penetration (and managed care more broadly) is critical given that the new delivery models are intended to improve population health outcomes and reduce the incidence of chronic diseases.

21. Ibrahim IA, Beich J, Sidorov J, Gabbay R, Yu L. Measuring outcomes of type 2 diabetes disease management program in an HMO setting. South Med J (2002) 95(1):78-87. doi:10.1097/00007611-200201000-00016

22. Rosenzweig JL, Taitel MS, Norman GK, Moore TJ, Turenne W, Tang P. Diabetes disease management in Medicare advantage reduces hospitalizations and costs. Am J Manag Care (2010) 16(7):e157-62.

23. Centers for Disease Control and Prevention. National Diabetes Fact Sheet: National Estimates and General Information on Diabetes and Prediabetes in the United States. (2011). Available from: http://www.cdc.gov/diabetes/pubs/pdf/ ndfs_2011.pdf

24. de Bruin SR, Heijink R, Lemmens LC, Struijs JN, Baan CA. Impact of disease management programs on healthcare expenditures for patients with diabetes, depression, heart failure or chronic obstructive pulmonary disease: a systematic review of the literature. Health Policy (2011) 101(2):105-21. doi:10.1016/j. healthpol.2011.03.006

25. Diabetes Prevention Program Research Group. The diabetes prevention program (DPP): description of lifestyle intervention. Diabetes Care (2002) 25(12):2165-71. doi:10.2337/diacare.25.12.2165

26. Dijkstra RF, Niessen LW, Braspenning JC, Adang E, Grol RT. Patient-centred and professional-directed implementation strategies for diabetes guidelines: a cluster-randomized trial-based cost-effectiveness analysis. Diabet Med (2006) 23(2):164-70. doi:10.1111/j.1464-5491.2005.01751.x

27. Eriksson KF, Lindgarde F. Prevention of type 2 (non-insulin-dependent) diabetes mellitus by diet and physical exercise. The 6-year Malmo feasibility study. Diabetologia (1991) 34(12):891-8. doi:10.1007/BF00400196

28. Hamman RF, Wing RR, Edelstein SL, Lachin JM, Bray GA, Delahanty L, et al. Effect of weight loss with lifestyle intervention on risk of diabetes. Diabetes Care (2006) 29(9):2102-7. doi:10.2337/dc06-0560

29. Knowler WC, Barrett-Connor E, Fowler SE, Hamman RF, Lachin JM, Walker $\mathrm{EA}$, et al. Reduction in the incidence of type 2 diabetes with lifestyle intervention or metformin. N Engl J Med (2002) 346(6):393-403. doi:10.1056/ NEJMoa012512

30. Mattke S, Seid M, Ma S. Evidence for the effect of disease management: is $\$ 1$ billion a year a good investment? Am J Manag Care (2007) 13(12): 670-6.

31. Agency for Healthcare Research and Quality. MEPS-HC Panel Design and Data Collection Process. (2013). Available from: http://meps.ahrq.gov/survey_comp/ hc_data_collection.jsp

32. Centers for Medicare and Medicaid Services. Medicare Advantage/Part D Contract and Enrollment Data, MA State/County Penetration. (2011). Available from: http://www.cms.gov/Research-Statistics-Data-and-Systems/ Statistics-Trends-and-Reports/MCRAdvPartDEnrolData/MA-State-CountyPenetration.html

33. Centers for Medicare and Medicaid Services. Health Plans, Reports, Files and Data: State County File, 2002-2005. (2013). Available from: http://www.cms.gov/Research-Statistics-Data-and-Systems/Statistics-Trendsand-Reports/HealthPlanRepFileData/SC.html

34. Nicklett EJ. Socioeconomic status and race/ethnicity independently predict health decline among older diabetics. BMC Public Health (2011) 11:684. doi: 10.1186/1471-2458-11-684

35. Shi L. The convergence of vulnerable characteristics and health insurance in the US. Soc Sci Med (2001) 53(4):519-29. doi:10.1016/S0277-9536(00)00357-9

36. Assendelft WJ, Nielen MM, Hettinga DM, van der Meer V, van Vliet M, Drenthen AJ, et al. Bridging the gap between public health and primary care in prevention of cardiometabolic diseases; background of and experiences with the prevention consultation in The Netherlands. Fam Pract (2012) 29(Suppl 1):i126-31. doi:10.1093/fampra/cmr120

37. Ng J, Scholle SH. Disparities in Quality of Care for Midlife Adults (Ages 45-64) Versus Older Adults (Ages >65). Prepared under Contract from the Agency for Healthcare Research and Quality and the Office of the Assistant Secretary for Planning and Evaluation, Under Contract No. HHSP233200800278A. Washington, DC: National Committee for Quality Assurance (2010). 
38. Lerman IG, Villa AR, Martinez CL, Cervantes Turrubiatez L, Aguilar Salinas CA, Wong B, et al. The prevalence of diabetes and associated coronary risk factors in urban and rural older Mexican populations. $J$ Am Geriatr Soc (1998) 46(11):1387-95. doi:10.1111/j.1532-5415.1998. tb06005.x

39. Rockwood K, Tan M, Phillips S, McDowell I. Prevalence of diabetes mellitus in elderly people in Canada: report from the Canadian Study of Health and Aging. Age Ageing (1998) 27(5):573-7. doi:10.1093/ageing/27.5.573

40. Kaiser Family Foundation. Medicare Advantage (MA) Plan Penetration. (2014). Available from: http://kff.org/other/state-indicator/medicareadvantage-penetration/
Conflict of Interest Statement: The authors declare that the research was conducted in the absence of any commercial or financial relationships that could be construed as a potential conflict of interest.

Copyright (c) 2015 Howard, Bernell, Wilmott, Casim, Wang, Pearson, Byler and Zhang. This is an open-access article distributed under the terms of the Creative Commons Attribution License (CC BY). The use, distribution or reproduction in other forums is permitted, provided the original author(s) or licensor are credited and that the original publication in this journal is cited, in accordance with accepted academic practice. No use, distribution or reproduction is permitted which does not comply with these terms. 